\title{
Promoção de competências de pensamento crítico em estudantes de enfermagem
}

\author{
Alice Mártires, Maria João Monteiro, \\ Conceição Rainho \& Maria Zita Castelo-Branco
}

\begin{abstract}
Resumo:
O pensamento crítico é, cada vez mais, essencial em todas as áreas de intervenção do ser humano, sendo fulcral na resolução de problemas e na tomada de decisão em enfermagem. No sentido de o desenvolver, a literatura evidencia a necessidade de investir em metodologias de aprendizagem ativa, nomeadamente a aprendizagem cooperativa. Neste contexto, procurámos com o presente estudo quantitativo, quasi-experimental, com desenho pré e pós-teste, avaliar o uso de grupos cooperativos na promoção de competências de pensamento crítico. Para a avaliação foi utilizado o Teste de Pensamento Crítico. A amostra não probabilística foi constituída por vinte e quatro estudantes, na sua maioria do sexo feminino, e com idades compreendidas entre os 18 e 33 anos. Os resultados evidenciaram diferenças estatisticamente significativas na competência análise, no que respeita à comparação sobre soluções apresentadas face a um problema ( $p=0.00$ ), e na competência avaliação, relativa à credibilidade de afirmações e robustez das relações inferenciais $(p=0.04)$. O uso de grupos cooperativos revelou-se importante na promoção de algumas das competências de pensamento crítico em observação, no entanto para consolidar esta hipótese é necessário a realização de intervenções e estudos mais prolongados.
\end{abstract}

Palavras-chave:

pensamento crítico; grupos cooperativos; enfermagem 


\title{
Use of cooperative groups in the promotion of critical thinking skills in nursing students
}

\begin{abstract}
Critical thinking is increasingly essential in all areas of human intervention, specially to problem solving and decision making in nursing. The literature shows the need to invest in active learning methodologies, namely cooperative learning, in order to develop critical thinking. In this context, we intend with this quantitative, quasi-experimental study, with pre and post-test design, to evaluate the use of cooperative groups in the promotion of critical thinking skills. For the evaluation was applied the Critical Thinking Test. The non-probabilistic sample consisted of twentyfour nursing students, mostly female and aged between 18 to 33 years. The results shown statistically significant differences in the analysis skill, regarding the comparison on solutions presented for a problem $(p=0.00)$, and in the evaluation skill, concerning the credibility of affirmations and robustness of inferential relationships $(p=0.04)$. The use of cooperative groups has proved to be important in promoting some of the critical thinking skills in observation. However, to reinforce this hypothesis it is necessary to carry out longer interventions and research studies.
\end{abstract}

Keywords: critical thinking; cooperative groups; nursing.

\section{Utilisation de groupes cooperatifs dans la promotion de la pensée critique chez l'étudiant des soins infirmiers}

Résumé: La pensée critique est de plus en plus essentielle dans tous les domaines de l'intervention humaine, car elle est au cœur de la résolution de problèmes et de la prise de décision en soins infirmiers. La littérature scientifique montre la nécessité d'investir dans des méthodologies d'apprentissage actif, à savoir un apprentissage coopératif, afin de développer la pensée critique. Dans ce contexte, avec cette étude quantitative quasi expérimentale, conception pré et post-test, nous avons l'intention d'évaluer l'utilisation de groupes coopératifs dans la promotion des capacités de pensée critique. Pour l’évaluation on a utilisé le Test de Pensée Critique. L'échantillon non probabiliste comprenait vingt quatre étudiants d'infirmière, la plupart étaient filles et âgés de 18 à 33 ans. Les résultats ont mis en évidence des différences statistiquement significatives dans la compétence analyse, par rapport à la comparaison des solutions présentées à un problème $(p=0,00)$, et dans la compétence évaluation, en ce qui concerne la crédibilité des affirmations et la solidité des relations inférentielles $(p=0,04)$. L'utilisation de groupes coopératifs s'est révélée importante dans la promotion de certaines compétences de pensée critique en observation, mais pour consolider cette hypothèse, il est nécessaire de mener des interventions et des études plus longues.

Mots-clés: pensée critique; groupes coopératifs; infirmier.

\section{Uso de grupos cooperativos en la promoción de competencias de pensamiento crítico en estudiantes de enfermería}

Resumen: El pensamiento crítico es, cada vez más, esencial en todas las áreas de intervención del ser humano, siendo fundamental en la resolución de problemas y en la toma de decisión en enfermería. En el sentido de desarroIlarlo, la literatura científica evidencia la necesidad de invertir en metodologías de aprendizaje activo, en particular el aprendizaje cooperativo. En este contexto, buscamos con el presente estudio cuantitativo, cuasi-experimental, con diseño pre y post-test, evaluar el uso de grupos cooperativos en la promoción de competencias de pensamiento crítico. Para la evaluación se utilizó la Prueba de Pensamiento Crítico. La muestra no probabilística fue constituida por veinticuatro estudiantes, en su mayoría, del sexo femenino, y con edades comprendidas entre los 18 y 33 años. Los resultados evidenciaron diferencias estadísticamente significativas en la competencia de análisis, en lo que se refiere a la comparación sobre soluciones presentadas frente a un problema $(p=0.00)$, y en la competencia evaluación, relativa a la credibilidad de afirmaciones y robustez de las relaciones inferenciales $(p=0.04)$. El uso de grupos cooperativos se reveló importante en la promoción de alguna de las competencias de pensamiento crítico en observación, pero para consolidar esta hipótesis es necesario la realización de intervenciones y estudios más prolongados.

Palabras clave: pensamiento crítico; grupos cooperativos; enfermería. 


\section{Introdução}

A crescente complexidade dos cuidados de saúde, bem como a evolução da disciplina de enfermagem, tem vindo a exigir que os estudantes desenvolvam cada vez mais competências e disposições de pensamento crítico. Os desafios da saúde e da doença no século XXI, requerem profissionais críticos e reflexivos, capazes de incluir na prática clínica uma nova visão integradora do cuidado humano.

A Ordem dos Enfermeiros (OE), enquanto associação profissional de direito público, tem como desígnio regular e controlar o exercício da enfermagem, defendendo a qualidade dos cuidados. Estes tomam como principal foco de atenção a promoção dos projetos de saúde que cada pessoa vive e persegue. A finalidade dos cuidados de enfermagem é prevenir a doença e promover os processos de readaptação, a satisfação das necessidades humanas fundamentais e a máxima independência na realização das atividades de vida. Em 2003, a OE definiu o perfil de competências do enfermeiro de cuidados gerais, agrupadas por áreas, relacionadas com os domínios de intervenção da enfermagem. De entre as diferentes competências associadas às áreas da prestação e gestão de cuidados, encontram-se as seguintes: (i) "aplica o pensamento crítico e as técnicas de resolução de problemas"; (ii) "ajuíza e toma decisões fundamentadas, qualquer que seja o contexto da prestação de cuidados" e (iii) "interpreta, de forma adequada, os dados objetivos e subjetivos, bem como os seus significados, tendo em vista uma prestação de cuidados segura" (Ordem dos Enfermeiros, 2012, p.19). A formação em enfermagem, que em Portugal se integra no Ensino Superior Politécnico, visa assegurar aos estudantes, o desenvolvimento de competências de pensamento crítico, para o exercício da profissão, norteada pela excelência e padrões de qualidade. Um dos desafios que se coloca aos professores do ensino de enfermagem é possibilitar aos estudantes a apropriação da melhor evidência científica e o desenvolvimento de competências de pensamento crítico, recorrendo a metodologias de aprendizagem ativa, a fim de promover a qualidade do processo de ensino e de aprendizagem, bem como a qualidade dos cuidados de enfermagem (Quadros \& Colomé, 2016). Neste enquadramento, os professores de enfermagem são corresponsáveis pelo desenvolvimento do pensamento crítico dos estudantes, de modo a promover nos mesmos competências e disposições que permitam a tomada de decisão, caracterizada pela complexidade e singularidade dos processos, o que coloca ao exercício da profissão elevados níveis de exigência científica, ética, cultural e humana.

Desde a década de noventa que a problematização do pensamento crítico em enfermagem tem sido objeto de preocupação, de forma dar resposta às mudanças que caracterizam os ambientes de prestação de cuidados de saúde, exigindo o compromisso com estratégias de ensino e aprendizagem ativas. Assim, este estudo teve como questão norteadora "Qual o impacto do uso de grupos cooperativos no desenvolvimento de 
competências de pensamento crítico em estudantes de enfermagem?". Com o mesmo pretendemos avaliar em que medida o uso de grupos cooperativos promoveu o desenvolvimento de competências de pensamento crítico num grupo de estudantes do curso de licenciatura em enfermagem de uma Universidade do Norte de Portugal, no decorrer da unidade curricular do $2^{\circ}$ ano e $2^{\circ}$ semestre - "Opção II - Gestão da Doença Crónica".

\section{Pensamento Crítico em Enfermagem}

Apesar de não existir uma definição universalmente aceite de pensamento crítico, é consensual que se trata de um processo de julgamento intencional e autorregulado, que resulta na interpretação, análise, avaliação e inferência, bem como na explicação das evidências, considerações conceptuais, metodológicas, contextuais ou de critérios em função do julgamento (Facione, 2015). O pensamento crítico é fundamental para os estudantes quando aprendem uns com os outros, sendo que o desenvolvimento de competências e disposições de pensamento crítico está associado ao sucesso académico, permitindo o pensar sobre o pensar, isto é, a metacognição (Facione \& Facione, 2007; Halpern, 2014).

A enfermagem é uma disciplina e uma profissão em que os enfermeiros precisam de integrar e desenvolver competências de pensamento crítico, nomeadamente para o julgamento clinico e tomada de decisão na resolução de problemas de saúde. Alfaro-LeFevre (2014), considera que o pensamento crítico é uma habilidade que os enfermeiros devem desenvolver para realizarem um raciocínio cuidadoso e deliberado, com foco nos resultados e fundamentado nas necessidades da pessoa. O mesmo é entendido como um processo de resolução de problemas que direciona para a tomada de decisão. O pensamento crítico é a base para o julgamento preciso e disciplinado, nas situações clínicas e em todas as etapas do processo de enfermagem. Este desenvolve-se em diferentes fases: avaliação ou apreciação inicial, identificação de diagnóstico de enfermagem, planeamento e implementação das intervenções de enfermagem, e avaliação dos resultados obtidos. As mesmas orientam o pensamento e a ação dos enfermeiros, segundo o que é exigido pelos diferentes padrões que orientam a prática profissional. Assim, em contexto formativo, as estratégias ativas de ensino e de aprendizagem são fundamentais, para a promoção de competências de pensamento critico nos estudantes, no sentido de estes poderem analisar criticamente as situações de saúde e planear, executar e avaliar intervenções de enfermagem significativas e adequadas às necessidades de cada pessoa (Alfaro-LeFevre, 2014).

\section{Competências e Disposições de Pensamento Crítico em Enfermagem}

Diversos autores têm procurado identificar competências e disposições de pensamento crítico, atitudes ou tendências para atuar de uma forma crítica (Tenreiro-Vieira \& Vieira, 2000). Em enfermagem e na prática profissional, competências e disposições 
de pensamento crítico são especialmente importantes, salientando-se: i) nos cuidados centrados nas pessoas saudáveis ou com doença, no respeito pelos seus valores e necessidades; ii) no trabalho em equipa intra e interprofissional, no respeito mútuo pelos saberes e tomada de decisão partilhados; iii) na prática profissional baseada em evidência para cuidados de excelência; iv) na melhoria da qualidade e segurança dos cuidados de saúde (Alfaro-LeFevre, 2014).

Reportando-nos a Watson (2002), pioneira na ciência do cuidar, a prática do cuidado é central à enfermagem e pressupõe a interação entre enfermeiro e pessoa, num ambiente de colaboração, respeito mútuo e confiança. Alfaro-LeFevre (2014), propõe o modelo dos quatro círculos, identificando as capacidades de pensamento crítico em enfermagem que esquematicamente se apresentam na Figura 1:

1. Atitudes e comportamentos de pensamento crítico, que se traduzem em ser curioso e inquisitivo, lógico e intuitivo, confiante e resiliente, honesto, responsável, cuidadoso, criativo, flexível, sensível à diversidade;

2. Conhecimentos teóricos e experienciais, habilidades e competências intelectuais, nomeadamente conhecimentos de anatomia, fisiologia e fisiopatologia, princípios éticos e legais da profissão de enfermagem, controlo de infeções, conhecimentos sobre o funcionamento biológico, psicológico, social, cultural e espiritual;

3. Habilidades e competências interpessoais, nomeadamente escuta ativa e empática, aceitar a expressão de sentimentos, ser confiável, admitir o erro, responder às preocupações do outro;

4. Habilidades e competências técnicas, nomeadamente procedimentos técnicos como punção venosa, intubação nasogástrica, execução de pensos, algaliação, administração de terapêutica, entre outros.

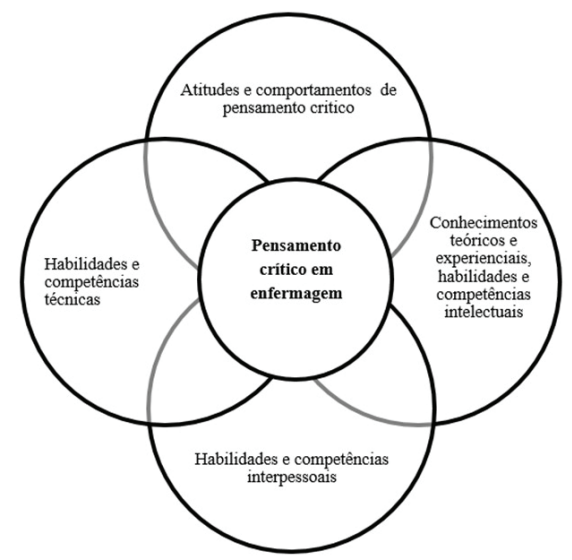

Figura 1 - Modelo dos quatro círculos do pensamento crítico em enfermagem. Adaptado de Alfaro-LeFevre (2014, p.59) 
O desenvolvimento de competências de pensamento crítico em enfermagem é complexo porque exige tempo, e ocorre com mais certeza através da experiência profissional. No entanto, como a segurança do utente constitui um dos aspetos centrais dos cuidados de qualidade, a utilização de experiências simuladas em sala de aula antes da aprendizagem em contexto clínico é, segundo a autora, uma forma excelente de desenvolver o pensamento crítico (Alfaro-LeFevre, 2014). Neste contexto, Billings e Halstead (2005) realçaram a importância da utilização de metodologias ativas no ensino superior, ajudando os estudantes a desenvolver competências de resolução de problemas, raciocínio crítico e analítico. Estas competências preparam-nos para tomar as decisões mais adequadas, para um maior envolvimento académico e, em última análise, melhores profissionais. Assim, a utilização de metodologias ativas de ensino e aprendizagem, aliada à promoção de ambientes facilitadores em sala de aula e no contexto da prática clinica, contribuem para que o estudante de enfermagem possa desenvolver competências e disposições de pensamento crítico.

\section{O Ensino e a Aprendizagem do Pensamento Critico em Enfermagem}

O professor de enfermagem pode utilizar diversas estratégias de aprendizagem ativa, com a finalidade de promover o pensamento crítico e facilitar a apropriação do conhecimento científico e tecnológico de enfermagem. Numa revisão integrativa da literatura sobre o conhecimento científico relativo ao pensamento crítico de estudantes de enfermagem, Peixoto e Peixoto (2017) concluíram que o pensamento crítico é um processo complexo e multidimensional e que é importante criar ambientes que estimulem a reflexão e a criatividade.

Diversas estratégias ativas de ensino e aprendizagem promotoras do desenvolvimento de pensamento crítico têm vindo a ser adotadas no ensino superior, de entre as quais se salientam: (i) a aprendizagem por questionamento, que implica o desenvolvimento da capacidade dos estudantes na formulação das suas próprias questões sobre o objeto de estudo e traduz-se na motivação, autonomia e no desenvolvimento da capacidade de raciocínio (Dillon, 1991); (ii) a aprendizagem baseada em estudos de caso, que se traduz na estratégia mais utilizada pelos professores de enfermagem na promoção do pensamento critico, visa promover o desenvolvimento de competências de tomada de decisão e auxilia o estudante com base em conhecimento prévio, a analisar um problema, fenómeno e situações clínicas concretas, para avaliar a situação, priorizar intervenções, analisar a evolução e validar as soluções possíveis (Galeano, Rossi \& Zago, 2003); (iii) a aprendizagem baseada em problemas, consisti num método de aprendizagem que utiliza problemas como ponto de partida para aquisição e integração de novos conhecimentos, desafiando os estudantes a comprometerem-se na procura do conhecimento ao equacionarem respostas e soluções (Barell, 2007); (iv) a aprendizagem cooperativa, que proporciona o desenvolvimento de capacidades 
intelectuais, interpessoais e sociais com benefícios para todos os elementos do grupo, em virtude da experiência de aprendizagem ser vivenciada em cooperação (Johnson, Johnson \& Smith, 1998).

No âmbito das estratégias de aprendizagem ativas, a aprendizagem cooperativa corresponde a uma abordagem de ensino que se apoia numa "metodologia com a qual os estudantes se ajudam no processo de aprendizagem, atuando como parceiros entre si e com o professor" (Lopes \& Silva, 2009, p.4). A mesma procura desenvolver nos estudantes a curiosidade científica, a capacidade de questionar evidências, a procura e discussão de soluções alternativas. Também na comunicação de opiniões e na revisão do próprio comportamento ou raciocínio, no sentido de contribuir para a formação de cidadãos, profissionais que irão intervir na sociedade de forma integradora. Neste sentido, os estudantes têm oportunidade de debater ideias, confrontar diferentes pontos de vista, avaliar a qualidade da informação e das soluções que propõem. De igual modo, desenvolvem capacidades para se tornarem melhores ouvintes, melhores comunicadores e melhores leitores. A aprendizagem cooperativa promove de forma significativa não só o desenvolvimento do pensamento crítico e criativo (Klimovienè, Urboniené \& Barzdziukiené, 2006; Wong, 2017), bem como as competências interpessoais, de comunicação, de liderança e de negociação (Damiani, 2008). A implementação da aprendizagem cooperativa na sala de aula obedece a determinadas fases que devem ser respeitadas. Assim, o professor assume um papel importante no enquadramento, organização e otimização do trabalho cooperativo. As tarefas estão relacionadas com as três fases distintas do processo: pré-implementação, implementação e pós-implementação (Lopes \& Silva, 2009, p.53). Esta estratégia permite a interação suportada num clima de abertura e confiança entre o professor e os estudantes e os estudantes entre si. Antes do início da atividade o professor procede à constituição dos grupos, onde os estudantes devem ser divididos em pequenos grupos ( 3 ou 4 elementos) heterogéneos, podendo para tal ser utilizados alguns critérios: rendimento académico, género, idade, etnia, motivação, capacidades e desempenho, de forma a potenciar o desempenho do grupo como um todo (Johnson e Johnson (2009).

Para utilizar os grupos cooperativos, Lopes e Silva (2009) afirmam ser necessário diferentes procedimentos: especificação dos objetivos da atividade; explicação da tarefa; supervisão da aprendizagem; apoio aos grupos para melhorar o desempenho interpessoal e grupal; e avaliação da aprendizagem e determinação, com os estudantes, do nível de eficácia de funcionamento do grupo. D’Souza, Venkatesaperumal, Radhakrishnan e Balachandran (2013) verificaram que a utilização de grupos cooperativos contribuiu para o desenvolvimento de competências de pensamento critico dos estudantes de enfermagem através da interação reciproca, diversidade de experiências e partilha de oportunidades de aprendizagem. Também Johnson e Johnson (2009) referem que os estudantes podem aprender ensinando e ensinar a aprender com os 
outros através de feedback. Os estudantes têm a oportunidade de conversar com os colegas, apresentar e defender ideias, trocar diferentes crenças, questionar outros modelos concetuais e estar ativamente envolvidos na sua aprendizagem (Srinivas, 2014).

\section{Metodologia}

Trata-se de um estudo quantitativo, do tipo quási-experimental, com desenho pré / pós-teste num só grupo de estudantes, com o objetivo de avaliar o impacto do uso de grupos cooperativos na promoção de competências de pensamento crítico. A amostra não probabilística foi constituída por vinte e quatro estudantes de uma turma do curso de licenciatura em enfermagem, sendo a maioria do sexo feminino e com idades compreendidas entre os 18 e 33 anos ( $M=20.87$; $D P=3.62)$. Este estudo desenvolveu-se no contexto da unidade curricular "Opção II - Gestão da Doença Crónica", do $2^{\circ}$ ano e $2^{\circ}$ semestre do ano letivo de 2017/2018. Os seguintes objetivos de aprendizagem foram definidos no âmbito desta unidade curricular: compreender a doença crónica e suas repercussões na pessoa e na família; analisar fatores que dificultam os processos de adesão aos regimes terapêuticos e gestão da doença crónica; identificar diagnósticos de enfermagem na pessoa com doença crónica e família; e planear intervenções de enfermagem orientadas para a pessoa com doença crónica e família.

Para a consecução dos objetivos foram constituídos grupos cooperativos (Lopes \& Silva, 2009), de forma a assegurar a partilha intensiva de informação, a troca de diferentes perspetivas e pontos de vista, e a análise de argumentos com vista à tomada de decisão clínica. A constituição dos grupos foi feita de forma aleatória e integrando 4 ou 5 elementos. Os grupos mantiveram-se durante o decurso da intervenção.

A metodologia utilizada foi desenvolvida em três momentos principais: i) primeiro momento - recorreu-se à análise de um artigo científico sobre a adesão ao tratamento anti-hipertensivo segundo dificuldades relatadas por indivíduos hipertensos; a mesma foi apoiada na grelha FRISCO adaptada por Payan-Carreira, Domingues, Monteiro e Rainho (2016), na discussão nos grupos cooperativos e partilha no grupo turma; ii) segundo momento - pesquisa bibliográfica sobre a gestão da doença crónica em doentes com diferentes tipos de patologia crónica, para identificação de diagnósticos de enfermagem, com discussão nos grupos cooperativos e partilha no grupo turma; iii) terceiro momento - pesquisa bibliográfica sobre a gestão da doença crónica em doentes com diferentes tipos de patologia crónica, para planeamento de intervenções de enfermagem, com discussão nos grupos cooperativos e partilha no grupo turma. 
Para a avaliação das competências de pensamento crítico foi aplicado, quer em pré-, quer em pós-teste, o Teste de Pensamento Crítico (TPC), de Lopes, Silva e Morais (2018), recentemente desenhado e validado para a população portuguesa. Este teste propõe a análise e resolução de uma situação da vida real, na qual se pode identificar um conjunto de circunstâncias problemáticas. Todos os estudantes responderam ao teste em contexto de sala de aula e na presença do docente, nos dois momentos previamente estabelecidos, garantindo o seu anonimato e consentimento livre e esclarecido. O preenchimento do TPC demorou em média 30 minutos. O mesmo é constituído por 6 questões de resposta aberta. A cotação das respostas é feita a partir de uma grelha de avaliação do TPC, numa escala de 0 (não identifica a ideia principal; não identifica nenhuma solução; não apresenta argumentos válidos; não apresenta pontos fracos da solução; não identifica informação pertinente; não responde) a 4 pontos (identifica a ideia principal; identifica as três soluções; argumenta de forma coerente; apresenta três ou mais pontos fracos; utiliza informação pertinente para melhorar os pontos fracos da solução escolhida; apresenta mais de duas soluções). Excecionalmente, a questão $2 \mathrm{~b}$ é a única que é cotada numa escala de 0 (não estabelece nenhuma comparação) a 3 (identifica duas ou mais diferenças). Dois avaliadores procederam à atribuição da pontuação nas dimensões de acordo com os critérios da grelha de avaliação. Após a recolha dos dados, o tratamento quantitativo foi realizado com o recurso ao Statistical Package for Social Sciences (SPSS), versão 22.0.

\section{Resultados}

Seguidamente, apresentam-se os resultados obtidos relativos ao pré- e pós-teste conforme consta da tabela 1. Como podemos verificar, na tabela 1 não se apresentam os resultados da pergunta $n .^{\circ} 6$, em virtude do número reduzido de respostas. Pelos resultados obtidos, de um modo geral, as médias relativas a quase todas as competências de pensamento crítico apresentaram valores mais elevados no pós-teste. Importa relevar que a sub-competência de comparação (pré-teste $=0.33$ e pós-teste=1.83) e a competência de explicação (pré-teste= 1.92 e pós-teste=2.08) foram aquelas que apresentaram valores médios mais elevados no pós-teste. Especificamente, e após aplicação do teste de Wilcoxon, que visa a comparação no mesmo grupo de sujeitos para analisar diferenças entre duas condições, pré e pós-teste, (Pestana \& Gageiro, 2005), para um nível de significância $p<0,05$, verificaram-se diferenças estatisticamente significativas na competência de análise, no que respeita à sub-competência de comparação $(p=0.00)$ e na competência de avaliação $(p=0.04)$. 
Tabela 1 - Resultados da avaliação do TPC em pré- e pós-teste

\begin{tabular}{lccccc}
\hline \multirow{2}{*}{ COMPETÊNCIAS } & \multicolumn{2}{c}{ PRÉ TESTE } & \multicolumn{2}{c}{ PÓS-TESTE } & \\
& $\mathrm{M}$ & $\mathrm{DP}$ & $\mathrm{M}$ & $\mathrm{DP}$ & $\mathrm{P}$ \\
\hline \hline 1. Interpretação & 1.38 & 1.05 & 1.79 & 1.10 & 0.14 \\
2. Análise & & & & & \\
2.a Identificação & 2.0 & 0.00 & 1.96 & 0.46 & 0.65 \\
2.b Comparação & 0.33 & 0.76 & 1.83 & 0.63 & 0.00 \\
3. Explicação & 1.92 & 0.28 & 2.08 & 0.40 & 0.10 \\
4. Avaliação & 1.38 & 0.64 & 1.83 & 0.70 & 0.04 \\
5. Síntese & 1.29 & 0.69 & 1.38 & 0.92 & 0.67 \\
\hline
\end{tabular}

$M=$ média; $D P=$ desvio padrão; $p=$ nível de significância

\section{Discussão e conclusões}

O estudo realizado visou avaliar o impacto do uso de grupos cooperativos no desenvolvimento de competências de pensamento crítico em estudantes de enfermagem. Foram verificadas melhorias na competência de análise, que pressupõe a identificação de relações intencionais e inferenciais entre afirmações, questões ou conceitos (Lopes, Silva \& Morais, 2018). No entanto, só na sub-competência de comparação foi evidente o melhor desempenho no pós-teste.

A competência de avaliação, que integra a capacidade para avaliar a credibilidade de afirmações e robustez lógica das relações inferenciais entre afirmações ou questões, demonstrou também um melhor desempenho no pós-teste. De facto, através da análise do artigo científico em questão com o uso da grelha FRISCO (primeiro momento da intervenção), ou da pesquisa bibliográfica (segundo e terceiro momento), em grupos cooperativos, foi possível aos estudantes desenvolverem processos de raciocínio que envolvem a análise e avaliação de inferências.

Estes resultados são consensuais com estudos anteriores, ao mostrar que os grupos cooperativos promovem a interação recíproca e a partilha de aprendizagens, permitindo que os estudantes se envolvam mais na aprendizagem e no desenvolvimento de competências de pensamento critico (Srinivas, 2014; D'Souza e outros, 2013; Johnson \& Johnson 2009; Barell, 2007). Também os estudos desenvolvidos por Khosravani, Manoochehri e Memarian (2004), e Gokhale (1995), concluíram que a utilização dos grupos cooperativos é favorável ao desenvolvimento de competências de pensamento crítico, através da discussão, esclarecimento e avaliação de ideias. 
Quanto às competências de interpretação, explicação, e síntese, apesar de apresentarem valores médios superiores no pós-teste, não se verificaram diferenças estatisticamente significativas. Como refere Alfaro-LeFevre (2014), o desenvolvimento de competências de pensamento crítico em enfermagem é complexo e exige tempo. Esta constatação coloca aos professores o desafio contínuo na utilização e avaliação de metodologias ativas de ensino e aprendizagem para o desenvolvimento do pensamento critico, nomeadamente na medida em que as mesmas podem ser desenhadas e implementadas no âmbito da formação em enfermagem.

No estudo desenvolvido, apesar das limitações encontradas, foi possível constatar o contributo de metodologias ativas de ensino e aprendizagem, concretamente o uso de grupos cooperativos, na promoção e desenvolvimento de competências de pensamento crítico em futuros enfermeiros. Estas competências são fundamentais para enfrentar as transformações operadas no ensino superior e no exercício da profissão de enfermagem. Pela qualificação científica, técnica e humana, os enfermeiros podem liderar o funcionamento do sistema de saúde e garantir o acesso das populações a cuidados de saúde de qualidade. A relevância destas metodologias é justificada no estudo de meta-análise de Abrami e outros (2015), cujos resultados sublinham o seu contributo para o ensino de competências e disposições de pensamento crítico de forma transversal a todos os níveis de ensino e áreas disciplinares. Assim, no âmbito futuro da unidade curricular "Gestão da Doença Crónica", prevê-se a continuidade da utilização dos grupos cooperativos e a introdução de outras metodologias ativas, nomeadamente a aprendizagem baseada em problemas.

\section{Limitações e trabalho futuro}

Os resultados obtidos evidenciarem a aquisição de competências de pensamento crítico nos estudantes de enfermagem, nomeadamente de análise e de avaliação. No entanto, é importante continuar a investir na implementação de metodologias ativas de ensino e aprendizagem ao longo das diferentes unidades curriculares do programa de estudos, para que de forma gradual ocorra o pleno desenvolvimento das diferentes competências de pensamento critico. O facto do estudo não ter utilizado um grupo de controlo, bem como da intervenção ter sido limitada em termos do seu tempo de aplicação, sugerimos a sua futura replicação com desenhos experimentais (i.e., com amostras aleatórias, grupo experimental e grupo de controlo) e segundo um intervalo de tempo mais prolongado. Será igualmente importante verificar o impacto do desenvolvimento de competências de pensamento crítico associado a outras variáveis (e.g. idade, sexo, desempenho académico, experiência profissional).

De salientar que o teste TPC utilizado integra apenas competências do domínio cognitivo, não tendo sido, por isso, possível avaliar as disposições de pensamento 
crítico dos estudantes. Assim, e atendendo à importância de promover igualmente disposições de pensamento crítico nos estudantes de enfermagem, trabalhos futuros deverão incluir instrumentos que permitam a sua avaliação.

\section{Agradecimentos}

Este trabalho insere-se no Projeto 'Pensamento Crítico nos Currículos do Ensino Superior Europeu - CRITHINKEDU', com o número de referência 2016-1-PT01-KA203-022808, financiado pela Comissão Europeia no âmbito do Programa Erasmus+. Coordenador: Professora Caroline Dominguez (UTAD/LabDCT-CIDTFF) - carold@utad.pt. Financiamento: 397,478.00€. O apoio da Comissão Europeia para a produção desta publicação não vincula esta entidade aos conteúdos que refletem apenas a visão dos seus autores, não sendo a Comissão responsável pela utilização que deles possa ser feita.

\section{Referências bibliográficas}

Abrami, P. C., Bernard, R. M., Borokhovski, E., Waddington, D. I., Wade, C. A., \& Persson, T. (2015). Strategies for teaching students to think critically: A meta-analysis. Review of Educational Research, 85(2), 275-314. doi:10.3102/0034654314551063.

Alfaro-LeFevre, R. (2014). Aplicação do processo de enfermagem. Fundamentos para o raciocínio clinico. Porto Alegre: Artmed.

Barell, J. (2007). Problem-based learning: An inquiry approach (2nd ed.). Thousand Oaks, CA: Corwin Press.

Billings, D. M. \& Halstead, J. A. (2005). Teaching in Nursing: A Guide for Faculty. Philadelphia, PA: W.B. Saunders.

Damiani, M. F. (2008). Entendendo o trabalho colaborativo em educação e revelando os seus benefícios. Educar em revista, 31, 213-230. doi.10.1590/S0104-40602008000100013.

Dillon, J. T. (1991). Questioning the use of questions. Journal of Educational Psychology, 83(1), 163-164.

D'Souza, M. S, Venkatesaperumal, R., Radhakrishnan, J. \& Balachandran, S. (2013) Engagement in clinical learning environment among nursing students: Role of nurse educators. Open Journal of Nursing, 3, 25-32. doi: 10.4236/ojn.2013.31004.

Facione, P. A. (2015). Critical thinking: What it is and why it counts. Millbrae, CA: Measured Reasons LLC. ISBN 13:978-1-891557-07-1.

Facione, P. A., \& Facione, N. C. (2007). Talking critical thinking. Change: The Magazine of Higher Learning, 39(2), 38-45. doi.10.3200/CHNG.39.2.38-45.

Fatareli, E. F., Ferreira, L. N. A., Ferreira, J. K. M. I. S., \& Queirós, S. L. (2010). Método cooperativo de aprendizagem Jigsaw no ensino de cinética química. Química Nova na Escola, 32(3), 161-168.

Galeano, L. E., Rossi, L. A., \& Zago, M. M. F. (2003). Roteiro instrucional para a elaboração de um estudo de caso clínico. Rev. Latino-am Enfermagem, 11(3), 371 - 5. 
Gokhale, A. A. (1995). Colaborative Learning Enhances Critical Thinking. Journal of Technology Education, 7(1), 22-30. doi: 10.21061/jte.v7i1.a.2.

Halpern, D. F. (2014). Thought and knowledge: An introduction to critical thinking (5th ed.). New York, NY: Psychology Press.

Johnson, D.W. \& Johnson, R.T. (2009). An Educational Psychology Success Story: Social Interdependence Theory and Cooperative Learning. Educational Researcher, 38(5), 365-379. doi: 10.3102/0013189X09339057

Johnson, D. W., Johnson, R. T. \& Smith, K. A. (1998). Cooperative Learning Returns To College What Evidence Is There That It Works? Change: The Magazine of Higher Learning, 30:4, 26-35. doi: 10.1080/00091389809602629.

Khosravani, S., Manoochehri, H., \& Memarian, R. (2004). Developing Critical Thinking Skills in Nursing Students by Group Dynamics. Journal of Advanced Nursing Practice, 7 (2), 1-9.

Klimovienè, G., Urboniené, J., \& Barzdziukiené, R. (2006). Developing critical thinking through cooperative learning. Kalbu studijos, 9, 77-84.

Lopes, J., \& Silva, H. S. (2009). A aprendizagem cooperativa na sala de aula: Um guia prático para o professor. Lisboa: Lidel.

Lopes, J., Silva, H., \& Morais, E. (2018). Teste de pensamento crítico para estudantes dos ensinos básico e secundário. Revista de Estudios e Investigación en Psicología y Educación, 5(2), 82-91. doi:10.17979/reipe.2018.5.2.3339.

Murray, D. E. \& Christinson, M. A. (2010). What English language teachers need to know. New York, NY: Routledge.

Ordem dos Enfermeiros. (2012). Regulamento do perfil de competências do enfermeiro de cuidados gerais. Lisboa: Autor.

Payan-Carreira, R., Dominguez, C., Monteiro, M. J., \& Rainho, S. P. M. C. (2016). Application of the ADAPTED FRISCO framework in case-based learning activities. Revista Lusófona de Educação, 32, 175-191. [Disponível em http://revistas.ulusofona.pt/index.php/rleducacao/article/view/5520, consultado em 17/09/2018].

Peixoto, T. \& Peixoto, N.(2016). Pensamento crítico dos estudantes de enfermagem em ensino clínico: uma revisão integrativa. Revista de Enfermagem Referência, 13,125-138.

Srinivas, H. (2015). Collaborative learning: 44 benefits of collaborative learning. [Disponivel em http:// www.gdrc.org/kmgmt/c-learn/44.html, consultado a 28/01/2019].

Quadros, J. S., \& Colomé, J. S. (2016). Metodologias de Ensino-Aprendizagem na Formação do Enfermeiro. Revista Baiana de Enfermagem, 30(2), 1-1-10. doi: 10.18471/rbe.v30i2.15662.

Tenreiro-Vieira, C. \& Vieira, R. (2000). Promover as capacidades de pensamento dos alunos: Tipos de Pensamento. Cadernos Interdisciplinares, 27, 16-21. 
Watson, J. (2002). Intentionality and Caring-Healing Consciousness: A practice of Transpersonal Nursing. Holistic Nursing Practice, 16(4), 12-19. doi:10.1097/00004650-200207000-00005.

Wong, M. (2017). A Cross-Sectional Study: Collaborative Learning Approach Enhances Learning Attitudes of Undergraduate Nursing Students. Journal of Nursing and Health Care, 5(1), 1-5.

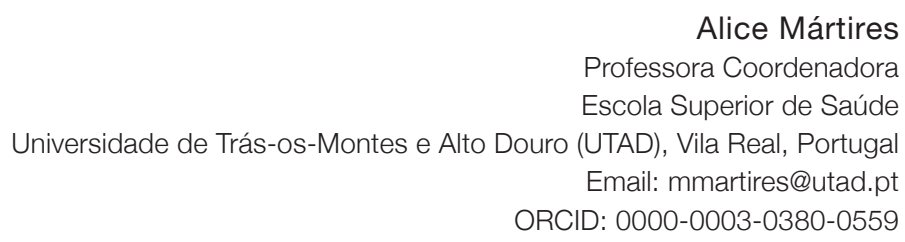

Maria João Monteiro

Professora Coordenadora

Escola Superior de Saúde

Universidade de Trás-os-Montes e Alto Douro (UTAD), Vila Real, Portugal

Centro de Investigação CINTESIS, Porto, Portugal

Email: mjmonteiro@utad.pt

ORCID: 0000-0003-0610-0670

Maria da Conceição Rainho

Professora Coordenadora

Escola Superior de Saúde

Universidade de Trás-os-Montes e Alto Douro (UTAD), Vila Real, Portugal

Email: crainho@utad.pt

ORCID: 0000-0002-3162-2086

Maria Zita Castelo Branco

Professora Adjunta

Escola Superior de Saúde

Universidade de Trás-os-Montes e Alto Douro (UTAD), Vila Real, Portugal

Email: mbranco@utad.pt

ORCID: 0000-003-3253-4739

Correspondência

Maria João Monteiro

Escola Superior de Saúde

Universidade de Trás-os-Montes e Alto Douro (UTAD), Vila Real, Portugal

Quinta de Prados

5000-801 Vila Real

Data de submissão: Setembro 2018

Data de avaliação: Novembro 2018

Date de publicação: Julho 2019 